\title{
The RAVE Survey: Constraining the Local Galactic Escape Speed
}

\section{Martin C. Smith, ${ }^{1}$ G. R. Ruchti, ${ }^{2}$ A. Helmi, ${ }^{1}$ R. F. G. Wyse ${ }^{2}$ and the RAVE collaboration}

${ }^{1}$ Kapteyn Astronomical Institute, University of Groningen, Groningen, The Netherlands

${ }^{2}$ Johns Hopkins University, Baltimore MD, USA

email: msmith@astro.rug.nl

\begin{abstract}
We report new constraints on the local escape speed of our Galaxy. Our analysis is based on a sample of high velocity stars from the RAVE survey and two previously published datasets (the Geneva-Copenhagen survey and the Beers et al. catalogue of metal-poor stars). We use cosmological simulations of disk galaxy formation to motivate our assumptions on the shape of the velocity distribution, allowing for a significantly more precise measurement of the escape velocity compared to previous studies. We find that the escape velocity lies within the range $492 \mathrm{~km} \mathrm{~s}^{-1}<v_{\text {esc }}<594 \mathrm{~km} \mathrm{~s}^{-1}$ (90\% confidence), with a median likelihood of $536 \mathrm{~km} \mathrm{~s}^{-1}$. The fact that $v_{\text {esc }}^{2}$ is significantly greater than $2 v_{\text {circ }}^{2}$ implies that there must be a significant amount of mass exterior to the Solar circle, i.e. this convincingly demonstrates the presence of a dark halo in the Galaxy. For a simple isothermal halo, one can calculate that the minimum radial extent is $\sim 54 \mathrm{kpc}$. We use our constraints on $v_{\text {esc }}$ to determine the mass of the Milky Way halo for three halo profiles. For example, an adiabatically contracted NFW halo model results in a virial mass of $1.31_{-0.49}^{+0.97} \times 10^{12} M_{\odot}$ and virial radius of $297_{-44}^{+60} \mathrm{kpc}(90 \%$ confidence). For this model the circular velocity at the virial radius is $141_{-19}^{+27} \mathrm{~km} \mathrm{~s}^{-1}$. Although our halo masses are model dependent, we find that they are in good agreement with each other.
\end{abstract}
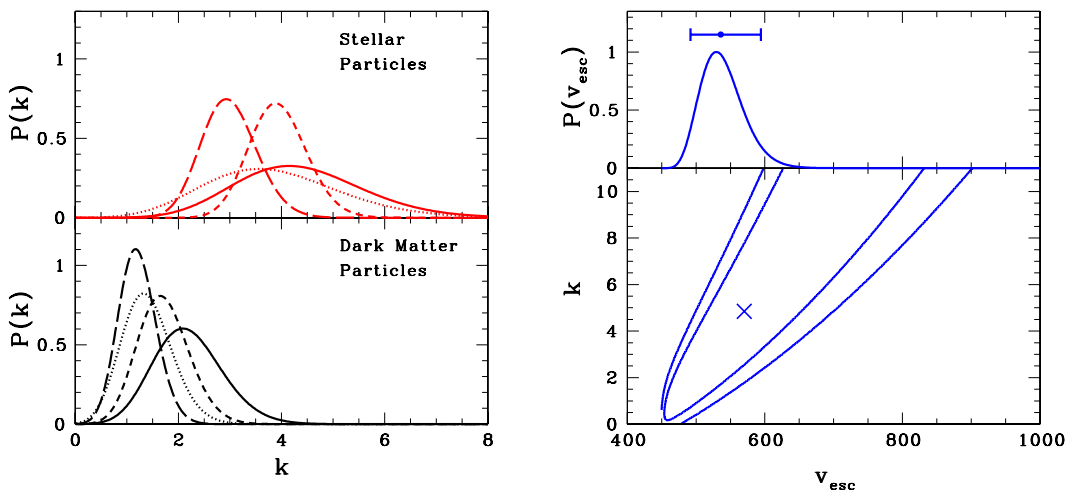

Figure 1. The left-hand panel shows the likelihood estimate for $k$ from four simulated galaxies, where $k$ denotes the shape of the tail of the velocity distribution. From this we deduce a uniform prior for $k \in[2.7,4.7]$. The right-hand panel shows the final likelihood contours for our observed high velocity stars. We also show the resulting constraints on $v_{\text {esc }}$ after applying our prior on $k$.

\section{Acknowledgements}

Funding for RAVE (www.rave-survey.org) has been provided by institutions of the RAVE participants and by their national funding agencies. 